Results Eleven HIV diagnoses occurred among 520 MSM with 652 person-years (PY) follow-up; HIV incidence was 1.7/ 100PY (95\%CI: 0.8-3.0), and highest among Hispanic MSM (2.6/100PY) and MSM aged $<30$ years (2.2/100PY). Median time to HIV diagnosis was 178 days (range 41-410). During follow-up, 18\% (94/520) received PEP again (range 1-4 times), with 58\% of PEP-repeaters (55/94) also given PrEP at some point. Of 460 MSM with SHC visits during follow-up, 202 were linked to or self-reported PrEP use, 200 were not linked to PrEP, and 58 declined navigation for PrEP. Of the 202 who linked to/self-reported PrEP use, 31 (15\%) had subsequent PEP event(s) at SHC. Eight of 279 MSM without evidence of PEP/PrEP during follow-up were diagnosed with HIV (incidence $=2.3 / 100 \mathrm{PY}$ ); NNT $=61$.

Conclusion Despite PrEP availability, fewer than half of PEP patients took up PrEP and a substantial proportion took PEP repeatedly; research to elucidate underlying reasons for PrEP and PEP use patterns (insurance/cost, convenience, self-perceived risk) is warranted.

Disclosure No significant relationships.

\section{P429 DOES HIV PRE-EXPOSURE PROPHYLAXIS (PREP) INITIATION IN SEXUAL HEALTH CLINICS IMPACT SUBSEQUENT HIV RISK?}

\footnotetext{
${ }^{1}$ Preeti Pathela*, ${ }^{2}$ Kelly Jamison, ${ }^{1}$ Christine Borges, ${ }^{3}$ Sarah Braunstein, ${ }^{3}$ Rachael Lazar, ${ }^{1}$ Susan Blank. 'New York City Department of Health and Mental Hygiene, Bureau of Sexually Transmitted Infections, New York City, USA; ${ }^{2}$ New York City Department of Health and Mental Hygiene, Bureau of STI, New York City, USA; ${ }^{3}$ New York City Department of Health and Mental Hygiene, New York City, USA
}

\subsection{6/sextrans-2019-sti.515}

Background Since late 2016, the PrEP program in New York City's 8 sexual health clinics (SHCs) has evolved from a model of active referral to external PrEP providers to on-site PrEP initiation (providing one month of PrEP and provider referral); this was implemented, clinic by clinic, over time. We hypothesized that HIV incidence would be lower for patients who received PrEP medication in SHCs.

Methods We matched the following groups of men-who-havesex-with-men (MSM) who received SHC PrEP services (12/ 2016-06/2017) to citywide HIV and STI registries: (1) sameday PrEP initiators, (2) referred-only for PrEP, (3) neverreferred (not interested in starting PrEP). We calculated HIV incidence after SHC PrEP service date, using HIV surveillance data through 06/30/2018. We assessed factors associated with HIV diagnosis using Cox models that included initiation vs referred-only status, linkage, age, race/ethnicity, incident STI (chlamydia/gonorrhea/primary or secondary syphilis), and 3month history of condomless receptive anal sex and number of sex partners.

Results There were 267 initiators, 607 referred-only, and 96 never-referred patients; $40 \%$ of both initiated and referredonly patients linked to PrEP providers. Of all MSM ( $n=970$; 1215 person-years [PY] of follow-up), 18 were newly diagnosed with HIV (median time to diagnosis 221 days; range 23-468). HIV incidence was 1.5/100PY (95\%CI 0.9-2.3) and not significantly different by group: $1.8 / 100 \mathrm{PY}$ among initiators (linked: 1.6, non-linked: 2.0); 1.3/100PY referred-only (linked: 0.0, non-linked: 2.2); 1.8/100PY never-referred. HIV risk did not differ for initiators versus referred-only patients, but was higher among non-linked than linked (aHR 5.6; 95\% CI 1.3-25.0) and those with STI versus no STI (aHR 10.1; 95\%CI 2.3-44.7).

Conclusion This examination of PrEP initiation in an episodic care setting did not find on-site PrEP provision to be associated with improved linkage and HIV outcomes. Understanding barriers to ongoing PrEP care is critical to improving linkage, and maximizing resources and the benefit of immediate PrEP access.

Disclosure No significant relationships.

\section{P430 PREDICTORS OF INTEREST IN SWITCHING FROM DAILY TO ON-DEMAND HIV PRE-EXPOSURE PROPHYLAXIS (PREP) AMONG AUSTRALIANS}

Vincent Cornelisse*, Luxi Lal, Brian Price, Edwina Wright. The Alfred Hospital, Department of Infectious Diseases, Melbourne, Australia

\subsection{6/sextrans-2019-sti.516}

Background The only HIV pre-exposure prophylaxis (PrEP) regimen approved in Australia is daily co-formulated tenofovir and emtricitabine. As an alternative, on-demand PrEP could offer benefits, including lower pill burden for people having sex infrequently. We surveyed PrEPX study participants to measure interest in switching from daily PrEP to on-demand PrEP.

Methods The survey asked 15 questions on demographics, sexual behaviour, interest in on-demand PrEP, and reasons for interest. Univariate regression analyses assessed associations between interest in on-demand PrEP and other survey questions, and questions with significant $(\mathrm{p}<0.1)$ association in univariate analysis were entered in a multivariate regression model.

Results 970 responses were complete. Respondents' median age was 39 years, and $99.6 \%$ were male. All had taken daily PrEP, but 14\% had ceased PrEP. 469 respondents (48\%; 95\% CI 45-52) reported interest in on-demand PrEP. In multivariate analysis, interest in on-demand PrEP was independently associated with having ceased PrEP (aOR 2.0, p<0.001), dissatisfaction with daily $\operatorname{PrEP}(\mathrm{aOR} 2.0, \mathrm{p}=0.027$ ), difficulty remembering to take pills every day (aOR 1.6, $\mathrm{p}=0.029)$, infrequently having sex that conferred HIV risk (aOR 3.7, $\mathrm{p}<0.001$ ), concerns about long term toxicity from PrEP (aOR 2.7, $\mathrm{p}<0.001$ ), and having no prior knowledge of on-demand PrEP (aOR 1.6, p<0.004). Respondents who were not interested in on-demand PrEP $(\mathrm{N}=501)$ reported concerns about its effectiveness (67\%), concerns about not remembering to take a dose at least 2 hours before sex (58\%), having unplanned sex (15\%), and having frequent sex and hence needing to take PrEP daily (2\%).

Conclusion This is the first study of interest in switching from daily to on-demand PrEP. Half of respondents were interested, and interest was most strongly associated with infrequency of sex and concerns about long-term toxicity. However, many respondents had concerns about the effectiveness of ondemand PrEP, and about forgetting to take on-demand PrEP at least two hours before sex.

Disclosure No significant relationships. 